\title{
Über die Frommann'schen Linien der Achsenzylinder der Nervenfasern.
}

\author{
Von \\ Dr. M. Noda und cand. med. G. Yasuzumi. \\ Aus dem Anatomischen Institut der Medizinischen Akademie zu Osaka \\ (Leiter: Prof. Dr. T. Tomita).
}

Mit 4 Textfiguren.

\section{Einleitung.}

Zum ersten Male berichtet C. Frommann im Jahre 1864 über querstreifige Strukturen in den Achsenzylindern bei der Silberfärbung bei seiner Arbeit „,Zur Silberfärbung der Axenzylinder". Diese durch die Silberfärbung hervorgerufenen querstreifigen Strukturen der Achsenzylinder wurden dann nach ihrem Entdecker als die Frommann'schen Linien bezeichnet. Seit der Entdeckung derselben sind sehr viele Publikationen darüber erschienen. Jedoch, ob diese Struktur ein eigentümlicher Bau oder ein Kunstprodukt ist, ist noch Sache der Diskussion.

Retzius (1910) hat aber ganz deutlich eine spiralige Anordnung an den markhaltigen Nervenfasern aus dem Trigeminus erwachsener Haie gefunden und behauptet: Die Regelmässigkeit in der Anordnung der spiraligen Bänder und die verhältmässig reine Färbung derselben sprechen nicht für Kunstprodukte.

Es ist also noch eine Frage, ob die querstreifige und spiralige Struktur, die durch Silberlösung gefärbt wird, dem Lies egang'schen Ringe gleich ist.

Die Beobachtung über die Eintrittstelle der Silberlösung in den Achsenzylindern ist bedeutungsvoll, aber wir sehen noch keine genügende Erklärung. 
Dies ist die zweite Frage, deren Beantwortung hier auch unternommen ist.

\section{Methode.}

Bevor wir unsere eigene Methode beschreiben, möchten wir auf die Methode der verschiedenen Forscher über die Frommann'sche Linie einen Rückblick werfen. Eine Querstreifung in den Achsenzylindern der Nervenfasern und in den anderen verschiedenen Geweben konnten Arndt (1879) mit Gold, Palladium, Osmium, Karmin oder einfacher Härtung mit chromsaurem Ammoniak, Kuhnt (1877) nach Behandlung mit essigsaurem Bleioxyd und Schwefelkalium, Beer (1893) mit Eisenchlorid-dinitroresorcin, S. Meyer (1889) mit Methylenblau und nachfolgender Fixierung mit seiner Pikrinsäureglyzerinmischung, Joseph mit Gemisch von 10\% iger Salpetersäure und 10\% iger Silbernitrat zu gleichen Teileu und Nachhärtung in Kaliumbichromat, A. Fischel (1893) mit Mischung von 25 Teilen Ameisensäure, 25 Teilen aqua dest. und 50 Teilen 1\% iger Silbernitrat in vier bis sechs Tage und Retzius (1909) mit gewöhnlicher Versilberung (Arg. Nitr. 1:500 dest. Wasser) darstellen.

Als Untersuchungsmaterial haben wir den Nervus ischiadicus von Meerschweinchen, Frosch und Kaninchen verwendet. Die Versilberungsmethode wurde von uns nach dem unten beschriebenen Verfahren durchgeführt: Man lege die Nerven in ca. $20 \mathrm{ccm} \mathrm{n} / 10$ Silbernitratlösung 1-4 Tage im Dunkelzimmer. Dann spüle man sie einige Minuten lang in destilliertem Wasser. Nun zerzupfe man den Nerven, bedecke ihn dann mit einem Deckglase und setze an den Rand desselben einen kleinen Tropfen dünnen Glyzerins. Lässt man aber das Präparat einige Stunden im Tageslichte liegen, so erhielt die sog. Fromman'schen Linien.

Das Problem über die Spiralen und Eintrittsstelle von Silbernitrat wurden von uns kolloidchemisch in Proberöhrchen und Schale durchgeführt.

\section{Ergebnisse.}

1. Anordnung der Querlinien in den Achsenzylindern von Frosch, Kaninchen und Meerschweinchen.

Mit Hilfe der Inmersion erkennt man, dass die Körnchen nicht nur in den Querscheiben, sondern auch in den hellen Zwischenräumen zwischen diesen sich vorfinden, dass sie ferner den ganzen Achsenzylinder ausfüllen und niemals den Achsenzylinder als Ringe umgreifen. Das 
eigentümliche Bild der Querstreifung kommt also dadurch zustande, dass schwarze Körnchen in einem Medium so angeordnet sind, dass sie stellenweise sehr dicht, in den Zwischenräumen dagegen äusserst spärlich beisammen liegen. In den näheren Teile der Eintritts-

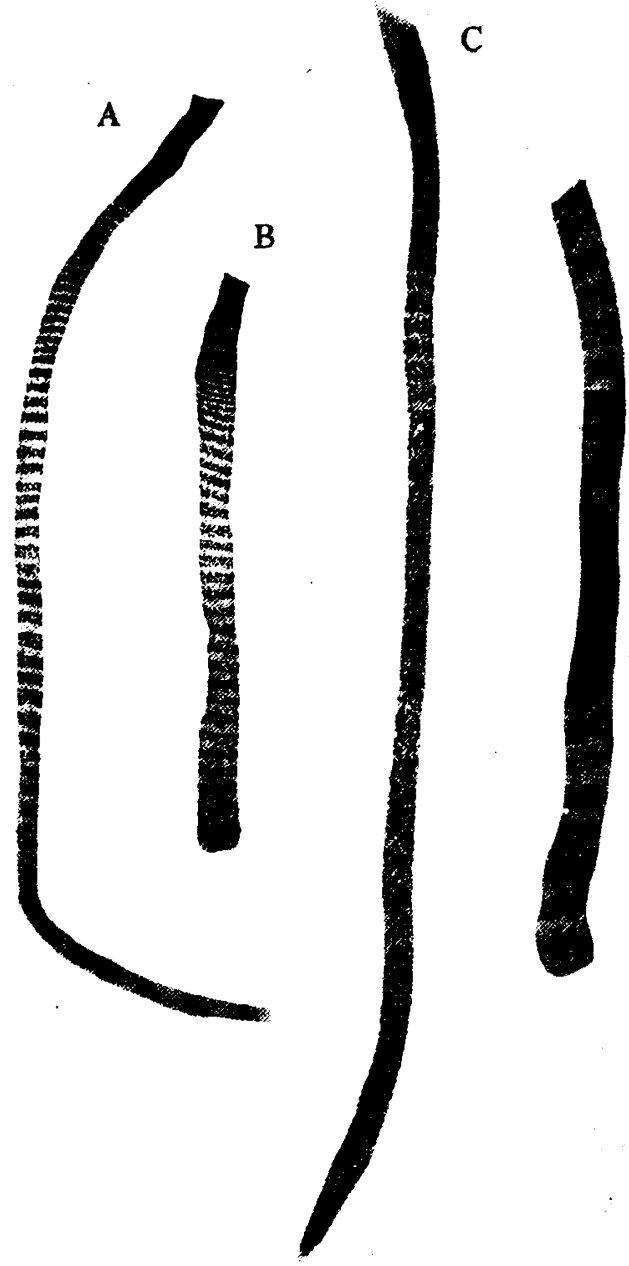

Fig. 1.

Frommann'sche Linien: A: bei Kaninchen. B: bei Kaninchen. C: bei Meerschweinchen. D : bei Frosch. stelle der Silberlösung (s. Über die Eintrittsstelle der Silberlösung in den Achsenzylindern) sind die Breite der Querstreifung und Zwischenräume sehr eng, aber in den entfernteren Teilen allmählich grösser. (Fig. 1.) Demnach ist die Zahl derselben abhängig von dem Diffusionsabstand des Silberuitrats.

Also : Die querstreifige Struktur, die durch Silberlösung gefärbt wird, ist wahrscheinlich dem Liesegang'schen Ring gleichen. Es ist daher von besonderem Interesse, die Gesetzmässigkeit mathematisch zu erklären.

2. Mathematische Erklärung.

Ist die Analogie zwischen Liesegang'schem Ring und Frommann'sche Linie oberflächlich, oder hat sie eimen wissenschaftlichen Grund? Um diese Frage zu beantworten, haben wir die Abstände der Frommann'schen Linien gemessen.

Bevor wir unsere eigenen Messungszahlen beschreiben, möchten wir auf frühere Angaben über die Diffusion des Silbernitrats und anderer 
Stoffe eiven Rückblick werfen. In Bezug auf die rhythmischen Niederschläge von Chrom- oder Chlorsilber gibt es zahlreiche Literatur. Die Ersten, welche die rhythmische Erscheinung in mathemathische Form $\mathrm{zu}$ bringen suchten und durch Messung die aufgestellten Gleichungen zu bestätigen suchen, waren Morse und Pierce (1903).

$$
\begin{aligned}
& \qquad \frac{h}{\sqrt{t}}=K, \quad \text { nach Morse und Pierce } \\
& \quad \frac{h}{\sqrt{t}}=a t+b, \text { nach M. Watanabe (1928) } \\
& h=\text { Deffusionsabstand } \\
& t=\text { Diffusionszeit } \\
& a \text { und } b \text { Konstante }
\end{aligned}
$$$$
t=\text { Diffusionszeit }
$$

Der Mitverfasser dieser Arbeit, G. Yasuzumi (1930) hat folgende Gleichung für die Diffusionsgeschwindigkeit von verschiedenen Fixierungsmitteln in einer Gelatinegallerte und irn Agar-Agar in Röhrchen aufgestellt :

$$
\begin{array}{ll}
R=a+b T & c=\text { elektrischer Widerstand vor der Diffusion } \\
R=c-a T^{b} & R=\text { elektrischer Widerstand } \\
R=c+\frac{T}{a+b T} & T=\text { Zeit } \\
R=a T^{-b} & a \text { und } b \text { Konstante }
\end{array}
$$

K. Jablczynski (1920) hat die Diffusionsgleichungen z. B. des Silbernitrats, in einer Gallerte abgeleitet.

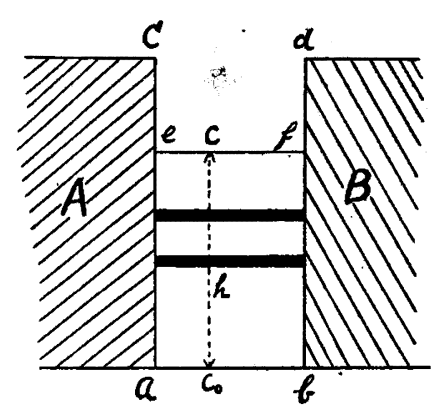

Fig. 2.

Diffusion von Silbernitrat in einer Chromgallerte. (nach $\mathbf{K}$. Jablczynski). $\frac{d h}{d t}=-\frac{K D Q_{0}}{Q} \frac{d c}{d h}$

$\frac{d h}{d t} \quad=$ Diffusionsgeschwindigkeit des Silbernitrats

$D \quad=$ Diffusionskoeffizienten desselben in Gelatine

$Q$ u. $Q_{o}=$ Querschnitte bei ef und $a b$ $\frac{d c}{d h}=\underset{\text { Konzentrationsabfall von Silber- }}{\text { nitrat }}$

C. u. $C_{o}=$ Konzentration von Silbernitrat

$\boldsymbol{K} \quad=$ Konstante

Bleibt der Querschnitt konstant $Q=Q_{0}$ und ist die Beobachtungsstrecke $h$ nicht zu lang, so können wir setzen : 


$$
\frac{d c}{d h}=\frac{C-C_{o}}{h-h_{o}}=-\frac{C_{o}}{h}
$$

Setzen wir den Wert $\frac{d c}{d h}$ von (2) in (1), so bekommen wir :

$$
\begin{aligned}
\frac{d h}{d t} & =\frac{K D C_{o}}{h} \\
\int_{0}^{h} h d h & =\int_{o}^{t} K D C_{o} d t \\
\frac{1}{2} h^{2} & =K D C_{o} t \quad \frac{h^{2}}{t}=2 K D C_{o}=K_{1}
\end{aligned}
$$

C. A. Schleussner (1924) hat die folgende Gleichung aufgestellt :

$$
\begin{aligned}
& \frac{X_{n}}{X_{n-1}}=K_{2} \\
& X_{n}=\text { Abstand des Ringes } \\
& n \text { =Ordnungszahl } \\
& K_{2}=\text { Konstante } \\
& \log X_{n}-\log X_{n-1}=K_{2} ; \\
& K=\Delta \log X \\
& \log X_{n}-\log X_{n-1}=K_{2} \text {; } \\
& \log X_{n-1}-\log X_{n-2}=K_{2} \text {; } \\
& \log X_{2}-\log X_{1}=K_{2} \\
& \log X_{1}-o \\
& \log X_{n}=K_{2} N \\
& N=\frac{\log X_{n}}{K_{2}} \\
& K_{1}=\frac{X_{2}}{t}=\text { Fick'sches Gesetz } \\
& \log X=\frac{\log K_{1}+\log t}{2} \\
& K_{2}=\frac{\log K_{1}+\log t}{2 N}
\end{aligned}
$$


Es ist unmöglich, mit den obigen Gleichungen direkt die Frommann'schen Linien zu messen, weil die Messung der Zeit und des Abstandes hierbei bedeutend schwieriger sind. C. A. Schleussner hat aber anstatt der direkten Messung des Abstandes die Anzahl ( $Z$ ) der Ringe (Liesegang'sche Linie) gezählt, die auf 10 Teile $(d)$ des Okularmikrometers kamen.

$$
Z=N_{x+d}-N_{x}
$$

Setzt man den Wert $N$ von (6) in diese Gleichung ein, so erhält man

$$
\begin{aligned}
& Z=\frac{1}{K_{2}}[\log (X+d)-\log X] \\
& Z=\frac{1}{K_{2}} \log \frac{X+d}{X}=\frac{1}{K_{2}} \log \left(1+\frac{d}{X}\right) \\
& K_{2}=\frac{\log \left(1+\frac{d}{X}\right)}{Z}
\end{aligned}
$$

Wir können bei der Messung der Frommann'schen Linien die Gleichung (9) direkt anwenden. In der Tabelle I. bringen wir die Messungen bei Kaninchen, Meerschweinchen und Frosch. 
Tabelle I.

Anordnungszahl der Frommann'schen Linien.

\begin{tabular}{|c|c|c|c|c|}
\hline Untersuchungsmaterial & $\boldsymbol{X}$ & $d$ & $Z$ & $K_{2}$ \\
\hline Kaninchen & $\begin{array}{cc}0.02 & \mathrm{~mm} \\
0.08 & \\
0.14 & \prime\end{array}$ & $\begin{array}{cc}0.06 & \mathrm{~mm} \\
0.06 & \\
0.06 & "\end{array}$ & $\begin{array}{r}17.0 \\
10.0 \\
8.7\end{array}$ & $\begin{array}{l}0.03542 \\
0.02430 \\
0.01991\end{array}$ \\
\hline Meerschweichen & $\begin{array}{ll}0.01 & " \\
0.03 & " \\
0.05 & " \\
0.07 & \text { " }\end{array}$ & $\begin{array}{ll}0.02 & " \\
0.02 & " \\
0.02 & " \\
0.02 & "\end{array}$ & $\begin{array}{l}8.4 \\
6.6 \\
5.0 \\
3.0\end{array}$ & $\begin{array}{l}0.05680 \\
0.03363 \\
0.02923 \\
0.03645\end{array}$ \\
\hline Frosch & $\begin{array}{l}0.250 \mathrm{~mm} \\
0.283 " \\
0.316 " \\
0.349 " \\
0.332 "\end{array}$ & $\begin{array}{l}0.033 \mathrm{~mm} \\
0.033 " \\
0.033 " \\
0.033 " \\
0.033 "\end{array}$ & $\begin{array}{l}20.0 \\
15.0 \\
13.0 \\
12.0 \\
11.0\end{array}$ & $\begin{array}{l}0.002692 \\
0.003204 \\
0.003317 \\
0.003271 \\
0.002272\end{array}$ \\
\hline Frosch & $\begin{array}{cc}0.01 & \mathrm{~mm} \\
0.05 & \prime \\
0.09 \quad "\end{array}$ & $\begin{array}{l}0.04 \\
0.04 \\
0.04\end{array}$ & $\begin{array}{r}J 0.0 \\
9.0 \\
7.0\end{array}$ & \\
\hline Kaninchen & $\begin{array}{l}0.01 \\
0.05 \\
0.09 \\
0.03\end{array}$ & $\begin{array}{l}0.04 \\
0.04 \\
0.04 \\
0.04\end{array}$ & $\begin{array}{r}23.0 \\
19.0 \\
16.0 \\
7.0\end{array}$ & \\
\hline Meerschweichen & $\begin{array}{l}0.01 \\
0.03 \\
0.05 \\
0.07\end{array}$ & $\begin{array}{ll}0.02 & " \\
0.02 & . \\
0.02 & " \\
0.02 & "\end{array}$ & $\begin{array}{l}8.0 \\
6.5 \\
5.5 \\
4.0\end{array}$ & \\
\hline
\end{tabular}

Vergleicht man die Werte der Tabellen untereinander, so sieht man, dass die Abstandskonstante $K_{2}$ nicht immer charakteristisch für die betreffende Querstreifung ist. Aber wir glauben, dass die Diffusion in den Acbsenzylindern etwas komplizierter als in Röhrchen ist. $K_{2}$ gibt daher Konstanz.

Das Resultat, dass die Zahl ziemlich langsam mit der Entfernung von der Eintrittsstelle des Silbernitrats absteigert, zeigt die Fromman'schen Linien ganz dasselbe Bild wie das in Röhrchen bieten.

3. Über die Retzius'schen Spiralringe.

Retzius (1910) hat deutlich eine spiralige Anordnung an den markhaltigen Nervenfasern gefunden und behauptet, dass es kein Kunstprodukt, sondern ganz ursprüngliche Struktur sei.

Wir haben die spiraligen Ringe in den Gelatinegallerten durch 
folgende Methode erhalten : Über die erstarrte Gelatinegallerte $20 \mathrm{ccm}$ $(10 \mathrm{Gr}$. Gelatine, $10 \mathrm{ccm}$. aq. dest. und $0,1 \mathrm{Gr}$. Bleiazetat) wird 0,5\% ige Kaliumbichromatlösung geschichtet. Nach 5-7 Tagen wird die, Lösung gegossen, und mit N/2 Silbernitrat anstatt der Kaliumbichromatlösung ersetzt, so traten an einigen Stellen. Ringe zusammen, und es bildet sich in den Ringen wahrscheinlich Löcher. Dieses Auseinanderbrechen und Zusammentreten der Ringe führt zur Bildung von Spiralen.

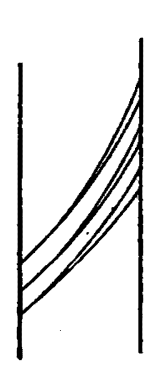

Fig. 3.

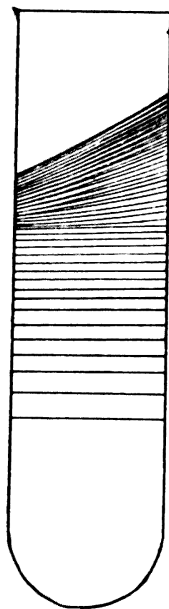

Schematische Darstellung der Diffusionsvorgänge von Silbernitrat in Gelatinegallerte, die eine schiefe Oberfläche hat.
Beim Versuche mit Chromsilber in Gelatine, wobei die Gelatine noch geringe Mengen von Blei enthiet, kommen die spiraligen Ringe im diffusen Tageslicht des Laboratoriums und auch im dunklen Zustande. Wenn die Oberfiäche der Gelatinegallerte schief ist, so kommt die Ringbildung wie Fig. 3., niemals die Spiralringe. Die Spiralen haben daher keinen Zusammenhang mit den Diffusionsflächen.

4. Über die Eintrittsstelle des Silbernitrats in den Achsenzylindern.

Durch mikroskopische Untersuchung finden wir, dass die Frommann'schen Linien an der Ranvier'schen Einschnürung und Schnittfläche des Nervenmarks beginnen. Es ist also keine Zweifel, dass das Silbernitrat von der Ranvier'schen

Einschnürung oder von den Rissstellen im Nerveumark und Schnittfläche desselben her eindringt. Sind auch andere Stellen, besonders SchmidtLantermann'schen Einkerbung Eintrittsstellen von Silbernitrat oder nicht? In Bezug auf die Schmidt-Lantermann'schen Einkerbungen sehen wir zahlreiche Literaturangaben.

Hennig, Töl, Rawitz, Kölliker, Wynn, Früst, und Chio halten sie für Kunstprodukte; aber folgende Forscher bezeichnen dieselben als präformierte Strukturen; Lantermann, Boll, Pelizzi, Rumpf, Rezonoco, Golgi, Ceni, Mendino, Cathani, Firelli, Margennhi, Villa, Perzik, Johanssen, Ranzier, Kuhnt, Boberi, Jacovi, Koch, Engelken, Schiefferdecker, Ramon y Cajal, Nemiloff, Wageotte u.a. Im Jahre 1926 hat F. Bito durch Kosaka augeregt gefunden, dass die Schmidt-Lantermann'schen Einkerbungen keine Artefakte, sondern eine eigentümliche Struktur in den peripheren markhaltigen Nervenfasern sind. 
Wir haben da indirekt das Problem : ,, Sind die Schmidt-Lantermann'schen Einkerbungen die Eintrittstelle des Silbernitrats oder nicht?" in einer chromgallerthaltigen Schale studiert. Setzt man jedoch einigen Tropfen Silbernitrat in Entfernung von einigen Zentimeteren auf die Chromgallerte, so entsteht ein Bild wie Fig. 4; man beobachtet eine helle, chromfreie oder chromarme Zone zwischen Chromsilberniederschlägen. Der Grund ist folgender : Durch Aufsetzen von Silbernitrat, durch Niederschlagsbildung, wird Kaliumbichromat entzogen. Sind num auf einer Chromgallerte zwei benachbarte Silbernitrattropfen

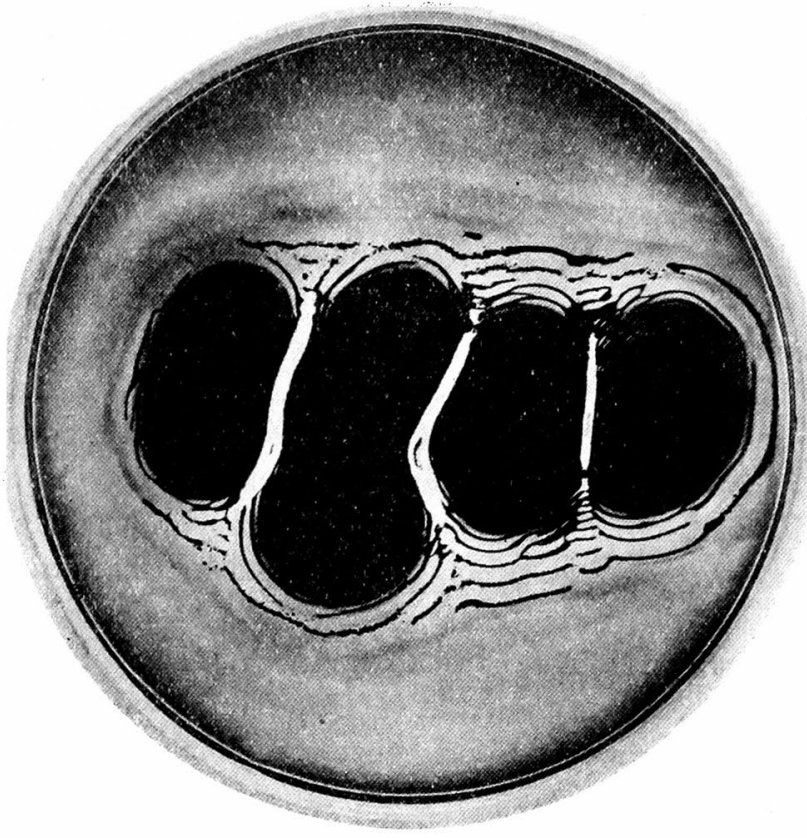

Fig. 4.

Heller, chromarmer oder freier Bezirk zwischen Silberniederschläge. aufgesetzt, : so bildet sich zwischen ihnen ein chromarmer oder chromfreier Bezirk.

Treten solche Niederschläge gleichzeitig an verschiedenen Stellen auf, so ist die Möglichkeit zur Entstehung der kompliziertesten Gebilde, unregelmässigen Anordnungen der Linien gegeben. Wir nehmen also als die Eintrittsstelle des Silbernitrats nur die Rissstelle im Nervenmark, d. h. die Ranvier'sche Einschnürungen und Schnittfläche des Nervenmarks an.

\section{Diskussion.}

Grandry (1869) kommt auf die früher von Frommann (1864) beschriebene Erscheinung der Querstreifung der Achsenzylinder nach Silberbehandlung zurück. Er behandelt Teile verschiedener nervöser Organe nach ausführlich beschriebener Technik möglichst bald nach dem Tode, wenigstens so früh, dass eine bemerkenswerte kadaveröse Verände- 
rung noch nicht anzunehmen ist; diesen Umstand hält er für wichtig. Grandry beschrieb die Erscheinung für die Achsenzylinder, im wesentlichen Frommanns Befunden entsprechend, er sagt, dass das Silbernitrat auf gewisse Teile des Achsenzylinders eine ,, action speciale" habe, andere allenfalls nur schwach angreife, und schliesst aus seinen Beobachtungen bezüglich des Baues des Achsenzylinders, dass er aus zwei physikalisch und chemisch differenten Substanzen, die vollkommen voneinander getrennt sind, bestehe, d.h. dass er wahrscheinlich von übereinander gelagerten Scheiben, die durch anders geartete Substanz getrennt sind, zusammengesetzt wird. Er sagt aber, dass noch weitere Untersuchungen angestellt werden müsst, " pour déterminer rigoureusement l'élément fondamental du cylindre de l'axe." Bezüglich der Nervenzellen und ihrer Fortsätze weichen seine Befunde von denen Frommanus ab, was wohl als Grund die verschiedene Technik hat. Während Frommann die Zellkörper gleichmässig braun gefärbt, die Kerne ungefärbt, die Zellfortsätze längsgestreift fand, aber keine Querstreifen beobachtete, kann Grandry eine sehr deutliche Querstreifung des Zellkörpers und der Fortsätze erkennen. Er schliesst aus seinen Wahrnehmungen bezüglich des Baues der Nervenzelle, dass sie aus zwei physikalisch und chemisch differenten Substanzen bestehe, d.h. dass auch hier vielleicht eine Anordnung in Scheiben vorliege, wie beim Achsenzylinder, und dass die Zellen und die Achsenzylinder nach Silberbehandlung unter gewissen Bedingungen die gleichen Charaktere bieten.

Hinsichtlich der Fromman'schen Linien waren A. Key und G. Retzius (1870) zu der Ansicht gekommen, die Querbänder nicht der eigentlichen, sondern einer Flächenschicht der Achsenzylinder angehören. Schiefferdecker (1887) kam zu dem Schlusse, das die Frommann'schen Linien in der von ihm beschriebenen, nicht als eigentliche Scheidenbildung aufzufassenden ,Gerinselscheide" liegen, sie stehen auch am Rande des Achsenzylinders immer über diesen etwas über. Lavdowsky (1876) nimint an, dass die nach Silberbehandlung auftretenden Querstreifen regelmässige Faltungen einer am Achsenzylinder existierenden Membran sind. Eine ,, rein physikalische "“ Erklärung versuchte Boveri (1885) zu geben. Für ihn besteht der Achsenzylinder aús Fibrillen, welche in einem mit lymphatischer Flüssigkeit erfüllten Räumen suspendiert sind, in welchem sich das Silber niederschlägt. In diesen Raum nun könne das Silbernitrat nur von der Ranvier'schen Einschuürung oder von der Rissstelle im Nervemmarke her eindringen, da es durch die unversehrte Markscheide nicht hindurchtreten kömne. 
J. Jakimovitch (1888) fand bei seiner Untersuchung bestätigt, dass man drei Typen der Querstreifung unterscheiden können :

1. Typus: Die hellen Linien sind breiter als die dunklen; letztere sind stark gekörnt-dieser Typus findet sich an Fasem oder Zellen, welche dern frisch getöteten Tiere entstammen.

2. Typus: Die Streifen sind gleich breit.

3. Typus: Die hellen Streifen sind schmaler als die dunkelndieser Typus findet sich an Präparaten von Objekten, die erst einige Zeit nach dem Tode dem Tiere entnommen werden.

Besonders aber hebt Jakimovitch hervor, dass die Form der Streifung wesentlich abhänge von dem Funktionszustande der Nervenfasern und Nervenzellen, dass sie überhaupt nicht auftrete an Nerven, welche vor dem Tode nicht funktionierten oder von Tieren stammen, die an Erschöpfung zu Grunde gegangen seien; oder von operierten Menschen, bei welchen die Narkose von langer Dauer gewesen sei.

In Told's Lehrbuch der Gewebelehre (1888), SchiefferdeckerKossel's Gewebelehre (1891), und Böhn-Davidoff's Lehrbuch der Histologie des Menschen (1895) finden wir noch nicht genügende Aufklärung über die Entstehung und Bedeutung derselben. In Krauses's Handbuch der menschlichen Anatomie (1876) ist auch eine Abbildung der fraglichen Querstreifen eingeführt. Kölliker (1896) macht folgende Betrachtung: Diese sogennanten Frommann'schen Linien rühren von Silberniederschlägen her, die zum Teil Achsenzylindern ihren Sitz haben, zum Teil auch einem Gerinsel, das zwischen dem Achsenzylinder und dem Marke in einem Raume auftritt, den Schiefferdecker als periaxialen Spaltraum bezeichnet und, für eimmal ohne bestimmte Beweise, als normal und mit einer minimalen Menge von Lymphe oder Ernährungsflüssigkeit erfüllt, betrachtet. In S. Ramon Cajal's grossem Werke ,,Textura del sistem nervioso del hombre y de los vertebredos", Tomo I (1899) finden sich auch Figuren von Nervenfasern des Frosches mit Frommann'schen Linien. In Huber's " A Textbook of Histology", (1900) können wir keine Erwähnung der fraglichen Linien finden. Obersteiner (1901) hat bei seiner Untersuchung behauptet, dass diese Linien aber an der Adventitien der Gefässe und anderen nicht nervösen Gewebe, selbst an Gelatinefäden dargestellt werden, also keineswegs etwas für den Achsenzylinder Charakteristisches ist. (Rabl, A. Fischel). Es handelt sich dabei nicht so sehr um eine histologische, also vielmehr um eine physikalische Erscheinung. In Quain's Elements of Anatomy, vol. 3. Neurology by Schäfer und Symington (1908) finden wir eine Abbildung der Querlinien, aber im Texte keine Erwähnung derselben. 
In der von Fürbringer umgearbeiteten Auflage von Gegenbauer's Lehrbuch der Anatomie des Menschen (I909) heisst es: disse Streifen sind nicht der Ausduck einer wirklichen Querstreifung, sondern ein Phänomen der Versilberung und werden auch an andern Geweben in ähnlichen Erscheinung durch Silberniederschläge hervorgerufen. L. v. Morochowetz (1878) glaubt, dass ,,um den Achsenzylinder gelegte ringförmige Raüme oder Kreiskanäle vorkommen, in deuen mit Silber sich schwärzende Stoffe liegen. Josepf (1890) nimmt an, dass der Achsenzylinder aus zwei Substanzen besteht, von welchen die eime homogen sei und Eigenschaft besitze, das Silber $\mathrm{zu}$ reduzieren. Der Achsenzylinder und die Ganglienzelle bestehen wach R. Arndt (1879) aus einer Grundsubstanz, in welcher kleine Kügelchen, die mit Silber sich schwarz färben, enthalten sind, und die er ,, Elementarkörperchen des Protoplasmas" oder ,, corpuscula primigenia protoplasmatis " nennt. Trifft nun ein Reiz den Achsenzylinder, so schwelleu die letzteren an, indem sie Flüssigkeit aus der Grundsubstanz aufnehmen; hierdurch wird diese dichter und zieht sich zusammen, besonders im dem Teile, welcher zwischen den reihenförmig geordneten Elementarkörperchen liegt. Indem nun die Elementarkörper näher aneinanderrücken und in gleicher Höhe gelagert erscheinen, bilden sie die Querstreifen. Arndt stellt sich weiter vor, dass-was übrings durch nichts bewiesen ist-von der Stelle des Reizes die Erregung der Elementarkörper, fortschreitend immer neue Querstreifen erzeugt und so als eine ,, Kontraktionswelle der Achsenzylinder mit der bekannten Geschwindigkeit ven $30-33 \mathrm{~m}$ in der Sekunde durcheilt““. Ganz dieselbe Einrichtung, welche auf ein ,, Bahn frei" (Arndt) herauskommen soll, findet sich in den Ganglienzellen. In weiter Verfolgung seiner Ansicht versteigt sich Arndt zu der Behauptung, dass die verschiedenen Temperamente der Menchen aus das verschiedene Mengenverhältniss zwischen Grumdsubstanz und Elementarkörperchen zurückzuführen seien! Morin (1890) spricht davon, dass Nerven und Muskeln denselben Bau sei. Altmann (1893) erwähnte, dass Silberkörnchen als Granula in dem bekannten Sinue seiner Theorie auffast, denen er das Geschäft der Nervenleitung überträgt,; dass er dem Achsenzylinder denselben Bau wie der Muskelfaser zuschreibt, jedoch dabei die Granula interfibrillär liegen lässt. A. Fischel ist im Jahre 1893 im seinem Werke ,,Zur Lehre von der Wirkung des Silbernitrats auf die Elemente des Nervensystems" zum folgeuden Schlusse gekommen, dass Querstreifen, die bei Behandlung mit Silbernitrat in den verschiedensten Gewebeelementen und Organen, besonders an Nervenfasern, Blutgefässen, an Knorpel etc. auftreten, Kunstprodukte sind, 
die dort erscheinen, wo kolloide Gebilde unter Einwirkung von Silbernitrat besonders unter gleichzeitiger Säurewirkung erstarren. Er erzeugte eine Querstreifung auch bei seiner nicht mehr funktionsfähigen Nervenfasern und hält es also für nicht berechtigt, dem funktionierenden Nerven allein die Pähigkeit zuzusprechen, nach Silberbehandlung quergestreift zu werden (Gegensatz zu Jakimovitch). Der Typus der Streifung steht nach Fischel in keiner Beziebung zum Zeitabstand zwischen Tod des Tieres und Entnahme des Objektes, dagegen bat die Art des Reagens Einfluss auf die Form der Querstreifung (Farbe und Grösse der Körnchen). Rabl (1893) nimmt auch wieder die Untersuchung der Frommann'schen Limien auf. Er geht auf die Natur des chemischen Prozesses bei der Behandlung der Gewebe mit Argentum nitricum ein und hält es neben der älteren Anschaung (Bildung eines Silberalbuminats) für möglich, dass sich das Silbersalz mit dern Alburnin durch Zusammenlagerung der Moleküle zu einem Silbernitrat-Eiweiss verbindet, analog jenem Vorgange, der sich bei Fällung des Harnstoffes aus seiner Lösung durch Quecksilbersalze abspielt. Er hält die kleinen Körnchen der Querstreifen bei den Frommann'schen Linien nicht für metallisches Silber, da sie sich in thioschwefelsaurem Natron lösen; dasselbe gilt auch von dem Silberniederschläge an den Endothelzellen der serösen Häute, der Blut- und Lymphgefässe, der Muskelfasern usw. Nach Rabl muss der Niederschlag vielmehr eine Verbindung des metallischen Silbers, wahrscheinlich ein Oxydationsprodukt desselben darstellen. Er findet nun, dass man die Querstreifen der Frommann'schen Linien auch an Bindegewebesbündeln hervorgerufen kann. Wie Jakimovitsch erhält er die Erzeugung der Querstreifen auch 24 Stunden nach dem Tode für möglich, auch bei Bindegewebesbündeln. Als Grundbedingung für das Gelingen der Methode betrachtet Rabl nicht die Intaktheit des Zellprotoplasmas-diese (Lebensfäfigkeit, Funktionsfäfigkeit) ist wohl mehr als 24 Stunden nach dem Tode nicht mehr anzunehmen-sondern die Anwesenheit noch gelösten unveränderten Eiweisses im Gewebe. Es ist unmöglich eine Querstreifung darzustellen, wenn die Stücke vorher der Einwirkung eines anderen Reagens unterworfen waren. Der Versuch im Reagensglas lehrt, dass das durch Säure oder Alkohol gefällte Eiweiss mit dem Silbernitrat keine durch Licht reduzierbare Verbindung eingeht, auch nach Auswaschen in fliessendem Wasser misslingt ein entsprechender Versuch. Rabl beschreibt genauer seine Feststellungen an seinen Präparaten. Er hat auch bei Knorpel Streifen ähnlicher Art dargestellt, sowie solche in der die Fettzellen umgebendem Flüssigkeit. Retzius (1910) hat aber gauz 
deutlich eine spiralige Anordnung an den markhaltigen Nervenfasern aus dem Trigeminus erwachsener Haie befunden und behauptet wie folgende: Die Regelmässigkeit in der Anordnung der spiraligen Bänder und die verhältmässig reine Färbung derselben sprechen nicht für Kunstprodukte. In Rauber-Kopsch's Lehrbuch der Anatomie des Menschen (12te Auflage, 1, 1913) sind die Frommann'schen Silberlinien erwähnt wie folgt: Der vom Silberniederschlag betreffende Achsenzylinder zeigt oft eine Querstreifung von abwechselnd einander folgenden hellen und dunkeln Streifen. Sie sind bekannt als Frommann'sche Linien. In Ph. Stöhr's Lehrbuch der Histologie (19te Auflage, 1922) finden sich auch Figuren von Nervenfasern des Frosches mit Frommann'schen Linien, obwohl nich so schön, als man sie erhalten kann. In Lehrbuch der Histologie und des Mikroskopischen Anatomie von Szymonowicz (1921) finden sich noch nicht genügende Aufklärung darüber. In ,, Die Kolloide in Biologie und Medizin von H. Bechhold (3te Auflage, 1920)" sind Frommann'schen Linien folgendermassen erwähnt: Bei Behandlung peripherer Nerven nach G. Golgi erhält man in den Achseuzylinder Schichtungen (Fromann'sche Linien), die sich als Artefakte erwiesen.

Aus Zusammenstellung von vorangegangene Angaben geht hervor, dass die Ansicht über die Beschaffenheit und Bedeutung der Fromman'schen Linien wechselnd gewesen ist. Folgende Forscher nennen dieselben ein Kunstprodukt: Schefferdecker, Boveri, Kölliker, Fürbringer, Joseph, A. Fischel und Bechhold. Im Gegensatz hierzu glauben andere zahlreiche Forscher, z.B. A. Key, Retzius, Labdowsky, Jakimonovitch, Morochowetz, R. Arndt, Morin, Altman und Andere, dass dieselben zu einer präformierten Struktur gehören.

Im allgemeineu scheint man aber immer mehr dazu geneigt $\mathrm{zu}$ sein, als ,,Kunstprodukte“ aufzufassen. Wir sehen genügende Einwände von A. Fischel gegen Jakimonovitch. Wir lassen die Einwände gegen Jakimonovitch unberücksichtigt. Wie Obersteiner schon beschrieben hat, handelt es sich bei den Frommann'schen Linien nicht so sehr um eine histologische, als viermehr um eine physikalische Erscheinung. An Gelatine und Nervenfasern haben wir ganz gleiche Resultate nicht erhalten. Daraus folgt, dass die Diffusion in den Achsenzylindern etwas komplizierter als in Röhrchen ist.

Nach unsern Befunde bei Achsenzylindern von Frosch, Meerschweinchen und Kaninchen sind die Frommann'schen Linien keine eigentliche Struktur, sondern die Silberniederschläge, die durch die 
bestimmten Diffusionsvorgängen des Silbernitrats hervorgerufen werden. Beweisgrunde dafür sind folgende :

1. Sie bestehen aus sehr zahlreichen Körnchen.

2. Die Körnchen finden sich nicht nur in den Querstreifen, sondern auch in den hellen -Zwischenräumen zwischen diesen.

3. Die Breite der Querstreifung und die Zahl derselben sind abhängig von dem Diffusionsabstand des Silbernitrats.

4. Gerade dieser dritte Beweis zeigt, dass sie ganz der rhythmischen Erscheinung des Silbernitrats in Röhrchen gleich sind.

Wir vermuten also, dass die Entstehung der Frommann'schen Linien und die rhythmische Fällung von Silberchromat in der Gelatine (Liesegang'sche Linie) auf gleicher Basis stehen. Verschiedene Faktoren wie Adsorption, Übersättigung, Diffusionswellen und Peptisation kommen ins Spiel bei den rhythmischen Niederschlägen von Silberchromat in Gelatine. Die obigen Faktoren sollen natürlich auch an den Achsenzylindern die sehr wichtige Rolle spielen. Die Entstehung dieser Linien ist offenbar durch die in Achsenzylindern vorhanden Chloride und andere Reduktionselemente des Silbernitrats bedingt.

Endlich müssen wir gegen Retzius einwenden, dass seine Behauptung unzutreffend ist, derzufolge die Spiralen nur an Nerven darstellbar sind.

Liesegang erhielt Spiralen von Silberchromat dadurch, dass er Silbernitrat in das in Gelatine verteilte Kaliumbichromat hineindiffundieren liess, wobei die Gelatine noch geringe Mengen von Säure und Gelatose enthielt.

Es ist die Ansicht geäussert werden, dass die Bildung von Spiralen anstatt der gewöhnlichen Form der Ringe eine Folge der Lichteinwirkung sei. Demgegenüber haben A. C. Chatterji und N. R. Dahr (1926) sowohl im diffusen Tageslicht des Laboratoriums wie im Dunkeln Spiralen erhalten von Zinkferrozyanid und Bleijodat in Gelatine, von Bleijodid in Kieselsäure, und von Mangansulfid, Bleichromat und Bleijodid in Agar. Ein ganz besonders interssanter Fall, spiraliger Ring wurde von T. Orlowski (1926) bei der Untersuchung der Einwirkung von Ammoniumhydroxydlösuug auf den sich beim Mischen von Gelatinesol mit Merkuronitratlösung bildenden weissen Niederschlag festgestellt, und zwar bei der Einwirkung auf das diesen Niederschlag enthaltende Gel in Reagenzgläsern. Küster (1916) wies darauf hin, dass im allgemeinen spiralige Ringe nur in Agar-Agar gebildet werden. Wir haben dagegen derartige Ringe nur in Gelatine erhalten. Hatscheck erhielt Spiralringe von Bleichromat dadurch, dass er eine 
Agargallerte mit 0,1 proz. Bleiazetat und einer geringen Beimengung von Essigsäure in ein grosses Volumen 0,5 prozentiger Kaliumbichromatlösung eintauchte. Tryhorn und Blaktin (1923) meinen, dass Licht ein wichtiger Faktor bei der Bildung dieser Ringe ist. A. C. Chatterji und N. R. Dahr (1926) haben aber im Laufe seiner Untersuchungen gefunden, dass Mangansulfid sowobl im Licht wie in der Dunkelbeit in Agargallerten spiralige Ringe ergibt. In gleicher Weise erhielten sie bei Bleijodid und Bleichromat in Agar und bei Bleijodat in Gelatine sowohl im Dunkelı wie im Lichte Ringe von spiralige Natur. Höchst wahrscheinlich kann Licht bei dieser anomalen Ringbildung des Bleichromats keine sehr grosse Rolle spielen. Spiralen werden ja hier auch im Lichte wie im Dunkeln gebildet, wie unsere und A. C. Chatterji und N. R. Dahr's Versuche zeigen. Über die Entstehung der Spiralen in Proberöhrchen nehmen A. C. Chatterji und N. R. Dahr folgendes an : Die Spiralringe werden dadurch hervogerufen, dass in einzelnen Teilen der Ringe, die von einem gallertigen Niederschlag gebildet werden, ein schnellere und unter günstigeren Bedingungen verlaufende Dehydration mit folgender Koagulation stattfindet als in der Hauptmasse der Ringe. Dies hat die Wirkung, dass die Struktur der Ringe uneinheitlich wird, und dass Elektrolyte durch einige der Poren frei diffundieren können, während die Hauptmasse der Ringe die Durchdiffusion von Elektrolyten nicht in merklichem Masse erlaubt. Hierdurch wird die Regelmässigkeit in der Bildung der Ringe zerstört. Eine notwendige Konsequenz der eben dargelegten Ansicht besteht darin, dass an verschiedenen Stellen sich Teile einer Spirale bilden, wenn die Dehydration oder Bildung von Löcher in den Ringen an zwei örtlich getrennten Punkten auftritt, wobei die dazwischen liegenden Teile der Ringe unverändert bleiben. In einem derartigen Falle werden diffundierende Elektrolyte durch die porōs gewordenen Stellen dringen, die eine gewisse Entfernung voneinander haben, und es wird eine Fällung an verschiedenen Stellen der unmittelbar unter dem Ring liegenden Schicht entstehen. Wemn die noch unversehrten dazwischen liegenden Teile der Ringe sich allmählich verändern und porös werden, so geht der diffundierende Elektrolyt auch hindurch unter Hervorrufen einer Fällung an den Stellen, die vorher klar waren. So werden die erst voneinander getrennten Teile der Spirale vereinigt. A. C. Chatterji und.N. R. Dahr sind da zu folgendem Schlusse gekommen: Bildung von Spiralen ist im Lichte wie im Dunkeh beobachtet worden. Sie tritt vor allem bei Salzen von schweren Metallen auf.

Wir haben anomale Ringe erhalten, die zwei Linien mit darstellen 
Über die Frommann'schen Iinien der Achsenzylinder der Nervenfasern.

aber später sich zu einer verbinden, wenn die Diffusionsfläche von Silbernitrat schief ist. Es ist noch $\mathrm{zu}$ bemerken, dass anomale Ringe init anderer als spiraliger Gestalt von Stuckert und von Riegel und Widgoff erhalten wurden.

Obige Tatsache liefern einen genügenden Beweis dafür, dass die Frommann'sche und Liesegang'sche Linien genetisch gleich sind, und dass die Retzius'schen Spiralen die Anomalie der Liesegang'schen Linien sind.

Hinsichtlich der Eintrittsstelle von Silbernitrat ist Boveri (1885) zu der Ansicht gekommen, dass Silbernitrat nur von der Ranvier'schen Einschnürung her eindringt. Simarro (1900) bespricht auch das Eindringen der Silberlösung in die Gewebsstücke, von der natürlichen Oberfläche aus scheint die Silberimprägnation hauptsächlich der Neuroglia zu folgen, deren Maschen intensiv schwarz erscheinen. An den Achsenzylindern dringt das Silber an den Ranvier'schen Einschnürungen ein, deren Scheiden sich klar und ohne Niederschläge färben. Die Markscheide bleibt ungefärbt, die Achsenzylinder werden imprägniert und erscheinen intensiv schwarz. Die Imprägnat ist dabei frei von Niederschlägen und es treten Fromman'sche Streifen und Ranvier' sche Scheiben auf. Wir behaupten durch unsere Befunde bei Achsenzylindern von Frosch, Meerschweinchen und Kaninchen und durch kolloidchemische Untersuchung im Chromgallerte (Die letzte Untersuchung sind von $R$. Liesegang $(1906,1910)$ im Chlornatriumgallerte studiert. Wir können aber keine ,,scheinbare chemische Anziehung“ beobachten) folgendes: Die Eintrittsstelle des Silbernitrats in Achsenzylindern sind Ranvier'sche Einschnürung und Schnittfläche des Nervenmarks, aber niemals Schmidt-Lantermann'sche Einkerbung.

\section{Zusammenfassung.}

1. Die Frommann'schen Linien sind nicht die eigentümlichen Strukturen der Achsenzylindern der Nervenfasern, sondern Kunstprodukte, weil sie genetisch ganz den Liesegang'schen Ringe gleich sind.

2. Die Spiralen nach Retzius sind auch Kunstprodukte, eine Anomalie der Liesegang'schen Ringe.

3. Die Eintrittsstelle des Silbernitrats sind Ranvier'sche Einschnürung und Schnittfläche des Nervenmarks.

Zum Schlusse möchten wir Herrn Prof. Dr. T. Tomita für die 
wertvollen Ratschläge zu dieser Arbeit unsern besten Dank aussprechen. Ausserdem danken wir Herrn Prof. J. Überschaar für seine Revision herzlichst.

\section{Literatur.}

1. Altman n, Arch. f. Anat. und Entwicklungsgesch. 1893.

2. Arndt, R., Etwas über die Achsenzylinder der Nervenfasern. Arch. f. pathol. Anat. und Phys. 1879.

3. Bechhold, H., Die Kolloide in Biologie und Medizin. 3te Auflage. 1922.

4. Beer. Th.. Jahrbücher f. Psychiatrie. 11. 1893.

5. Bito, F., Folia Anat. Japonica. Bd. 4.1920.

6. Boveri, Zeitschr. f. wiss. Mikrosk. Bd. 4. 1887.

7. Chatterji und Dahr, Koll. Zeitschr. Bd. 40. 1926.

8. Dagadkin, B., Koll. Zeitschr. Bd. 45.1928.

9. Engelmann, Arch. f. Phys. 1880.

10. Fischel, A., M. Schultze, Archiv. Bd. 42.1893.

11. Frommann, C., Virchow's Arch. Bd. 31. 1864.

12. Gierke, Zeitschr. f. wiss, Mikrosk. Bd. 1. 1889.

13. Hesse, Fr., Arch. f. Anat. und Phys. Anat. 1879.

14. Huber's A Textbook of Histology. 1900.

15. Jakimonovitch, J., Journ de l'anat. et de la phys. 1888.

16. Key, A., und Retzius, G., Studien in der Anatomie des Nervensystems und des Bindegegewebes. 2. H. 1. Abth. Stockholm 1876.

17. Klebs, Arch. f. Pathol. Anat. und Phys. Bd. 31.

18. Kölliker, sein Handbuch der Gewebelehre des Menschen. 1896.

19. Kuhnt, Arch. f. mikrosk. Anat. Bd. 13. 1877.

20. Khester, Koll. Zeitschr. Bd. 18. 1916.

21. Lavdowsky, Arch. f. mikrosk. Anat. Bd. 13.1876.

22. - Centralbl. f. Mediz. Wiss. 1876.

23. Liesegang, Zeitschr. f. Phys. Chem. 88. I.

24. —, Ann. d. Phys. Bd. 19. und Bd. 32.

25. Meyer, S., Zeitschr. f. wiss. Mikrosk. Bd. 6. 1889.

26. Rauber-Kopsch's Lehrbuch der Anatomie des Menschen. 12te Auflage, 1. 1913.

27. Rabl, Zeitschr. f. wiss. Mikrosk. Bd. 11. 1894.

28. Rawitz, B., Arch. f. Anat. und Entwicklungsgesch. 1879.

29. Retzius, Biochemische Untersuchungen von G. Retzius. Bd. 15. 1910.

30. Schiefferdecker, Arch. f. mikrosk. Anat. Bd. 30. 1887. und Bd. 31. 1888.

31. Schultze, Arch. f. Anat. und Entw. 1878.

32. Stadtmüller, Anat. Heite. :Bd. 59. 1921.

33. Simarro, Zeitschr. f. wiss. Mikrosk. Bd. 18.1901.

34. Stör's Lehrbuch der Histologie. 19. Auflage. 1922.

35. Stucker, Koll, Zeitschr. Bd. 37. 1925.

36. Szy monowitz, sein Lehrbuch der Histologie und der mikrosk. Anat. 4. 1921.

37. Widg off, Journ. Phys. Chem. Bd. 29. 1925.

38. Yasuzumi, Folia Anat. Japonica. Bd. 8. 1930. 